\title{
Role of Renin-Angiotensin System Components in Hypertension During Pregnancy
}

\author{
Xuan Jin ${ }^{1 *}$, Jing Zhang ${ }^{1}$, Yuanfang Zhu ${ }^{2}$, Pei Li ${ }^{1}$, GuiyingJin ${ }^{2}$ and Yu Zeng ${ }^{3}$ \\ ${ }^{1}$ Center for Assisted Reproduction, Affiliated Hospital of Nanchang University, Nanchang, Jiangxi, China \\ ${ }^{2}$ Department of Obstetrics and Gynecology, Affiliated Hospital of Nanchang University, Nanchang, Jiangxi, China
}

${ }^{3}$ Department of Surgery, Affiliated Hospital of Nanchang University, Nanchang, Jiangxi, China

*Corresponding author: Xuan Jin, Center for Assisted Reproduction, Affiliated Hospital of Nanchang University, Nanchang, Jiangxi, China

Submission: 眥 October 24, 2017; Published: 业July 02, 2018

\begin{abstract}
Background: Hypertension is common disease amongst women with pregnancy. It is associated with significant maternal, fetal and infant morbidity. Current research on the mechanism of the hypertensive disorders is limited. The renin-angiotensin system (RAS) is a hormone signaling cascade that regulates blood pressure and systemic electrolyte and fluid balance. Our study was designed to focus on the mechanism of gestational hypertension before preeclampsia occurred, exploring the change in both circulating RAS and local RAS.
\end{abstract}

Methods: The human kidney tissue were collected from patients suffered renal tumor (n: 5) or chronic renal insufficiency (n: 25). The human placental tissues were collected from woman with normal pregnancies (n: 35) and gestational hypertension (n: 21). There is no significant difference in maternal age between normal and gestational hypertension subjects. All the participants received written information, and signed the consent.

Results: Before delivery, the circulating PRL and Ang II concentration in the gestational hypertension is significantly lower than that in normal pregnancy. There is no significant difference between gestational hypertension and normal pregnancy after delivery. In the local RAS, AT1, Ang II and Ang 1-7 levels in gestational hypertension placenta were significantly higher than normal pregnancy. There is no statistical difference of renin, AGT, and ACE, ACE2 between the two subjects.

Conclusion: The change of Ang (1-7) expression in our study is different from the previous studies in preeclampsia. More attention should be put on the balance of Ang II and Ang (1-7).

Keywords: Gestational hypertension; Pregnancy; Renin-angiotensin system; Blood pressure; Vascular endothelial growth factor; cAMP responsive element binding protein

Abbreviations: RAS: Renin-Angiotensin System; AGT: Angiotensinogen; Ang-I: Antiotensin-I; ACE: Angiotensin-Converting Enzyme; Ang II: AngiotensinII; Ang (1-7): Antiotensin (1-7); CREB: cAMP Responsive Element Binding protein; BP: Blood Pressure; CRI: Chronic Renal Insufficiency; VEGF: Vascular Endothelial Growth Factor; AT1: Angiotensin II receptor type 1; PRL: Renin Activity

\section{Background}

The National High Blood Pressure Education Program of the NHLBI classifies hypertensive disorders of pregnancy into following categories: chronic hypertension, gestational hypertension, preeclampsia, preeclampsia superimposed on preexisting hypertension [1]. Hypertension in pregnancy is defined as $140 / 90 \mathrm{mmHg}$ or greater. Depending on the population studied, the incidence during pregnancy ranges from $0.5 \%$ to $3.0 \%$ [2]. Although the potential harmful effects of elevated blood pressure on both mother and fetus has been concerned, relatively little is known about its pathogenesis and prevention.

Preeclampsia is a disorder of pregnancy characterized by hypertension and proteinuria. Preeclampisa and its convulsive form, eclampisa, are associated with $10 \%$ to $25 \%$ of direct maternal deaths and stillbirths, neonatal morbidity and mortality in lowincome and middle-income countries [3]. The renin-angiotensin system (RAS) is a hormone signaling cascade that regulates blood pressure and systemic electrolyte and fluid balance. Regulation of the RAS in preeclampisa has been researched, different with uncomplicated pregnancy, in which most circulating RAS components increase, preeclamptic women have lower circulating levels of RAS components [4]. Whether the same case of the level of RAS components in preeclampsia happens in gestational hypertension patients, little study has been set.

Renin is synthesized and released by juxtaglomerular cells of the afferent renal arterioles, and cleaves the 452-amino acid 
angiotensinogen (AGT) to yield the 10-amino acid long peptide, antiotensin-I (Ang I). The biologically inactive Ang I is then cleaved by angiotensin-converting enzyme (ACE), made primarily in lung endothelium, to the biologically functional angiotensin-II (Ang II), eight-amino acid long effector molecule of the RAS [5]. The components of RAS are not unique to the kidney but are synthesized in many tissues. Placenta is one of the major local RAS during pregnancy [6]. Delivery of the placenta can usually resolve the acute clinical symptoms of preeclampsia, suggesting that the placenta plays a central role in preeclampsia pathogenesis. Is the alterations in tissue-specific RAS in placenta same with that detected by plasma components activity? As for local uteroplacental RAS, there are some research have been done, however the results are various and controversial. Shah et al. [7] demonstrated an increase in renin level in the deciduas vera of preeclamptic women. On the contrary, Herse et al. [8] argued that without any increase of other local RAS components observed in decidua of preeclamptic patients except angiotensin receptors AT1R. Anton et al. [9] reports in a clinical trial that AT1R mRNA and Ang II level were significantly higher in preeclampsia patients compared with normal pregnancies, despite a decrease in circulating Ang II. And Ang (1-7) concentrations decreased both in the uterus and placenta in the rat preeclampsia model [10]. Those suggest that it is necessary to conduct further investigation.

The mechanisms that govern the maintenance and reacquisition of the renin phenotype are not well understood. It has been shown that the cAMP pathway is crucial in the regulation of renin synthesis and release [11,12]. Phosphorylated form of cAMP responsive element binding protein (CREB) associated with its co-activators CBP and p300 mediated the transcriptional effect of cAMP by binding to the cAMP response element present in the upstream region of renin gene [13-16].

Gestational hypertension is elevated blood pressure and without proteinuria, which develops after 20 weeks of gestation in a previously normotensive woman. Approximately 15-45\% gestational hypertension will eventually develop preeclampsia $[17,18]$. Treatment on gestational hypertension can prevent

\section{RNA extraction and quantitative real-time PCR}

Table 1: Clinical profile of the pregant women. Values are expressed as means \pm SEMs

\begin{tabular}{|c|c|c|}
\hline Patient Clinical Characteristics & Normal Pregnancy & 21 \\
\hline N & 35 & $24.6 \pm 1.2$ \\
\hline Age (years) & $22.5 \pm 1.5$ & $37.5 \pm 0.9$ \\
\hline Gestational age (weeks) & $37.8 \pm 1.6$ & $155 \pm 8$ \\
\hline Systolic BP (mm Hg) & $128 \pm 11$ & $96 \pm 4$ \\
\hline Diastolic BP (mm Hg) & $78 \pm 6$ & None \\
\hline Proteinuria & None & None \\
\hline Number of patients reveiving & None & \\
\hline Antihypertensive medications & & \\
\hline
\end{tabular}


Tissues were ground in Tri-Reagent (Molecular Research Center, Cincinnati, OH). Total RNA was extracted according to the manufacturer's directions. Contaminating DNA was removed using the DNA-free kit (Ambion, Austin, TX). The cDNA was prepared from $2 \mu \mathrm{g}$ of RNA using Moloney murine leukemia virus reverse transcriptase (Life Technologies, Grand Island, NY) and an oligo (dT) 15 primer according to the manufacturer's directions. PCR was performed on $2 \mu \mathrm{l}$ of the RT reaction as a template using Taq DNA polymerase (Promega, Madison, WI). The primer sequences for all the genes examined are listed in (Table 1).

\section{Immuno histochemistry}

Serial $5 \mu \mathrm{m}$ sections of the tissue were deparaffinized and rehydrated through degraded ethanol. The sections were incubated with the primary antibodies specific for renin (Santa Cruz), angiotensin II receptor type 1 (AT1) (abcam), and CREB (abcam) respectively in $10 \%$ goat serum at room temperature for $2 \mathrm{hr}$, and then biotinylated secondary antibody and horseradish peroxidaseconjugated strepavidin, washing $3 \times 10 \mathrm{~min}$ after each incubating. Normal IgG instead of the primary antibody was served as the negative controls.

\section{Measurement of renin activity (PRL) and antiotensin II (Ang II)}

Circulating renin activity was measured by radioactive immunoassay with quantitative determination of angiotensin I. Blood samples were collected in EDTA containing tubes in ice and centrifuged at $2000 \mathrm{x}$ g for 10 minutes, $500 \mu$ supernatant of sample were harvested, and added with $10 \mu \mathrm{l}$ phenylmethysulfonyl fluoride and $50 \mu$ l angiotensin I buffer, after 90 minutes incubation, $50 \mu \mathrm{l}$ sample and $500 \mu \mathrm{l}$ tracers were added to the bottom of tubes which were coated with the 125I-labeled hormone, BSA, phosphate buffer, stabilizers, preservatives, and an inert red dye. After 3 hours incubation, the mixture was aspirated and a Gamma counter suitable for counting 125I measured the radioactivity of tubes. PRA was calculated as nanograms angiotensin I per milliliter per hour, and Ang II was calculated as picograms Ang II concentration in plasma as per milliliter.

\section{Western blot}

The tissues from various groups were homogenized in lysis buffer $(5 \mathrm{mmol} / \mathrm{L}$ phosphate buffer, pH 7.2, containing 0.1\% Triton $\mathrm{X}-100,1 \mathrm{mM}$ phenyl methyl sulfonyl fluoride, $1 \mathrm{mg} / \mathrm{L}$ chymostatin) respectively and the protein content of the supernatant after centrifugation was determined by spectrophotometer. The sample lysates were mixed with the loading buffer $(62.5 \mathrm{mM}$, 1,4-dithiothreitol, $5 \%$ sodium dodecyl sulfate (SDS), and $10 \%$ glycerol), boiled for 8min, separated by SDS-polyacrylamide gel electrophoresis $(30 \mu \mathrm{g}$ total protein/lane). After electrophoretic transferred to the polyvinylidene difluoride membrane, the membranes were blocked with $5 \%$ non fat milk/PBS for $1 \mathrm{~h}$, followed by incubation at $20^{\circ} \mathrm{C}$ for $1 \mathrm{hr}$ with the primary antibodies. Beta-actin or GAPDH was used as a loading control. The membranes were then washed for three times, 5 min for each, in 5\% milk/PBS and incubated with HRP-conjugated goat anti-rabbit IgG $(0.04 \mu \mathrm{g} /$ $\mathrm{ml}$ ) in $5 \%$ milk/PBS for $1 \mathrm{~h}$ respectively. The membranes were washed in PBS for three times, 5 min for each, followed by 5 min of incubation with SuperSignal $囚$ West Pico substrate, then subjected to x-ray autoradiography. For the negative controls, the primary antibodies were replaced by the normal IgG of the same origin.

\section{Competitive enzyme-linked immunosorbent assay (cELISA)}

The specificity of human tissue was tested by an indirect competitive ELISA. 96-well microplates (Nunc MaxiSorp, Roskilde, Denmark) were coated with the respective antigens by adding $100 \mu \mathrm{L}$ of the antigen solution $(2 \mu \mathrm{g} / \mathrm{mL})$ (cloud-clone corp.) in coating buffer (50mM sodium carbonate, $\mathrm{pH} 9.5$ ) and incubation overnight at $4{ }^{\circ} \mathrm{C}$. The plates were blocked for $30 \mathrm{~min}$ at room temperature (RT) with $1 \%$ bovine serum albumin (BSA) in PBS and then incubated with either serially diluted tissue sample or undiluted positive and negative controls for $1 \mathrm{~h}$ at RT. After three times washing with the provided washing buffer, the plates were incubated with HRP-labelled anti-human IgG (Bio-Rad) 1:5,000 in PBS-T or for $1 \mathrm{hr}$ at RT. The reaction was stopped by using $50 \mu \mathrm{l}$ of the stopping solution. The immune reaction was detected with TMB ready-to-use substrate (Sigma-Aldrich Co.). The optical density (OD) was measured at $450 \mathrm{~nm}$ in a microtiter plate reader (Tecan, Groedig, Austria). The test samples were considered negative if the optical density $\geq 50 \%$ of the mean of the negative controls.

\section{Statistical analysis}

Values shown are given as the mean \pm SEM. The data were analyzed using one-way ANOVA as appropriate. P-values $<0.05$ were considered statistically significant.

\section{Results}

\section{Blood pressure and plasma ras components level}

Table 1 shows the clinical profile of the pregnant women, 35 women with normal pregnancies and 21 women with gestational hypertension. The gestational hypertension subjects had hypertension as shown (mean blood pressure: 155/96mmHg versus $128 / 78 \mathrm{mmHg}$ ). None of the subjects had proteinuria. All subjects were matched for gestational age, and there is no significant difference in maternal age between normal and gestational hypertension subjects.

Figure 1 shows the plasma PRL and Ang II levels in the normal pregnancies and gestational hypertension. Before delivery, the PRL concentration in the gestational hypertension is significantly lower than that in normal pregnancy. After delivery, concentration of PRL in gestational hypertension increased, and that in normal pregnancy decreased a little, there is no significant difference between gestational hypertension and normal pregnancy after delivery. 

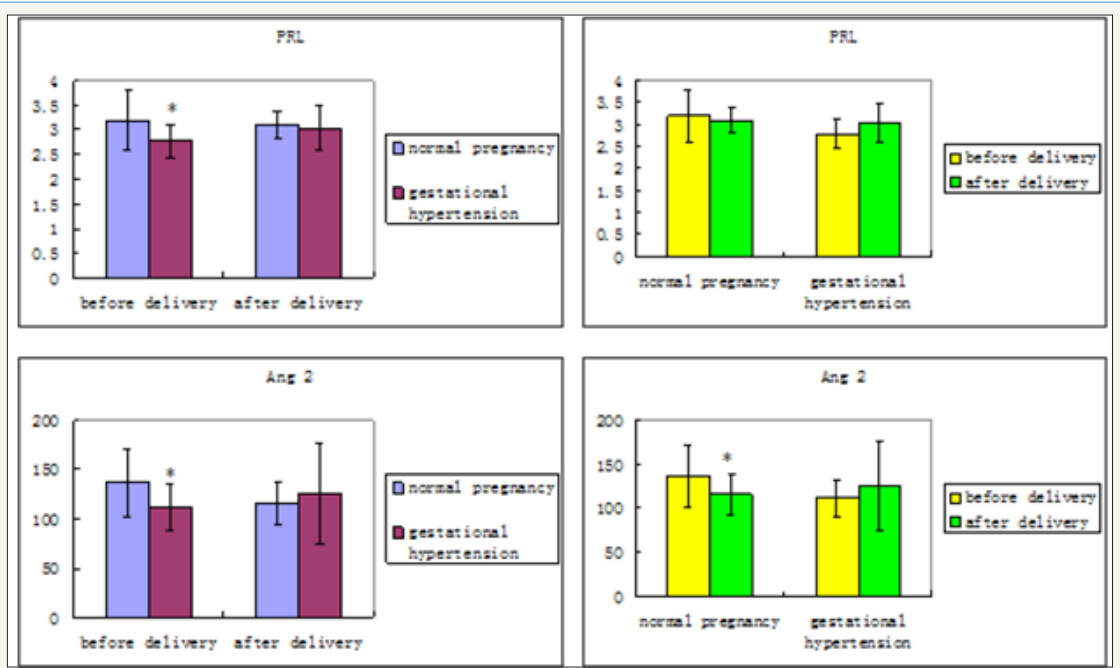

Figure 1: Circulating PRL and AngII levels in normal pregnancy and gestational hypertension

Values are expressed as means \pm SEMs. ${ }^{*} \mathrm{P}<0.05$

For Ang II, it is lower in gestational hypertension before delivery than that in normal pregnancy, and the difference is statistically significant. In the normal pregnancy, Ang II decreased significantly

\section{Ras components levels in normal and gestational hypertension placenta}

\begin{tabular}{|c|c|c|c|c|}
\hline & \multicolumn{2}{|c|}{ Normal Pregnancy } & \multicolumn{2}{|c|}{ Gestational Hypertension } \\
\hline & PRL & AngII & PRL & AngII \\
\hline Before delivery (1-2 days) & $3.19 \pm 0.61$ & $136.32 \pm 34.99$ & $2.78 \pm 0.33$ & $111.9 \pm 20.91$ \\
\hline After delivery (1-2 days) & $3.09 \pm 0.28$ & $115.88 \pm 23.15$ & $3.04 \pm 0.44$ & $125.7 \pm 51.39$ \\
\hline
\end{tabular}

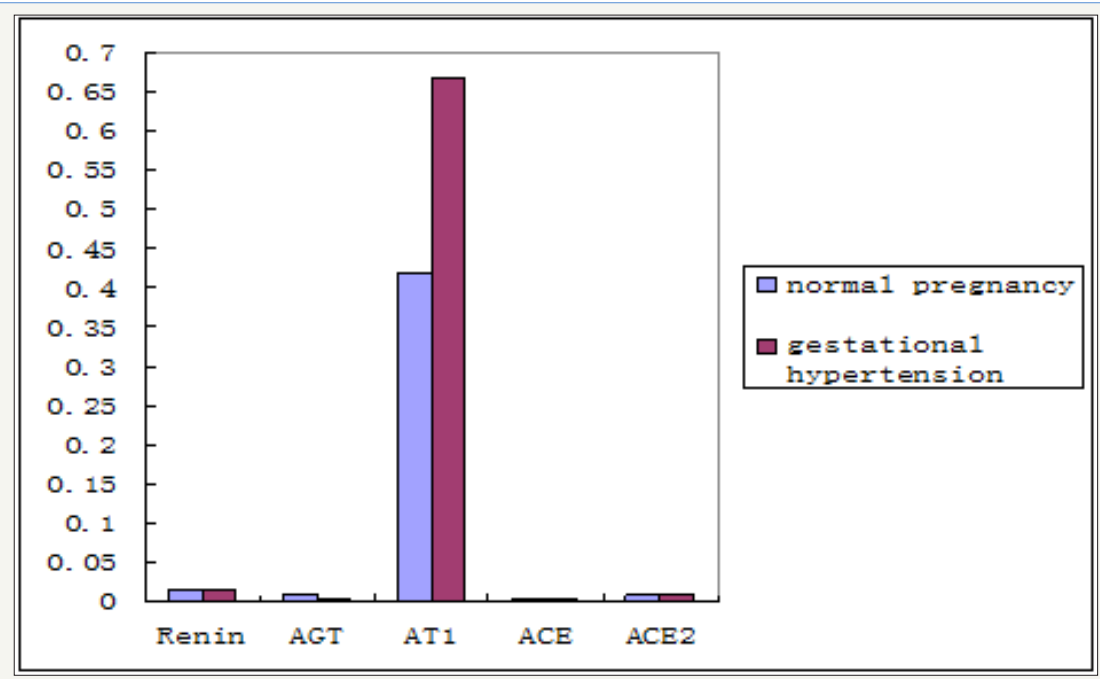

Figure 2a: Gene expression of RAS components in the placenta of normal pregnancy and gestational hypertension was determined. Values are expressed as means \pm SEMs. ${ }^{*} \mathrm{P}<0.05$, ${ }^{* *} \mathrm{P}<0.01$

RAS components mRNA were measured by reverse transcription, real-time PCR of renin, AGT, angiotensin II receptor 1 (AT1), ACE, ACE2.

RAS components include renin, Angiotensinogen (AGT), angiotensin II (Ang II), angiotensin 1-7 (Ang 1-7), and angiotensin II receptor (AT), angiotensin-converting enzyme (ACE). The expression of RAS components mRNA and protein was shown in Figure 1. There was no significant difference of mRNA in renin, AGT, AT1, ACE, and ACE2 between normal and hypertension subjects ( $>0.05)$, although there were trend for higher AT1 mRNA and lower AGT mRNA in gestational hypertension placenta versus normal, however, statistical significance was not reached (Figure 2a). Gestational hypertension placenta was found to have around 4 fold higher AT1 protein compared with normal pregnancy (0.53 \pm 0.05 versus $0.14 \pm 0.02, \mathrm{P}<0.01$ ) (Figure $2 \mathrm{~b}$ ). In addition, the proteins of Ang II and Ang 1-7 levels in gestational hypertension placenta were significantly higher than the concentrations found in 
tissue from normal pregnancy (Ang II: $38.15 \pm 5.03$ versus 20.02

were also determined, no statistical difference was found between $\pm 2.07, \mathrm{P}<0.01$; Ang 1-7: $15.44 \pm 2.03$ versus $11.63 \pm 1.83, \mathrm{P}<0.05$ ) the two subjects (Figure 3).

(Figure 2c). The proteins expression of renin, AGT, and ACE, ACE2

\begin{tabular}{|c|c|c|c|}
\hline Unit: E-04 & Renin & AGT & ACE \\
\hline Normal Pregnancy & $161 \pm 49$ & $91 \pm 13$ & $4188 \pm 1509$ \\
\hline Gestational hypertension & $159 \pm 51$ & $43 \pm 3$ & $6681 \pm 2833$ \\
\hline
\end{tabular}

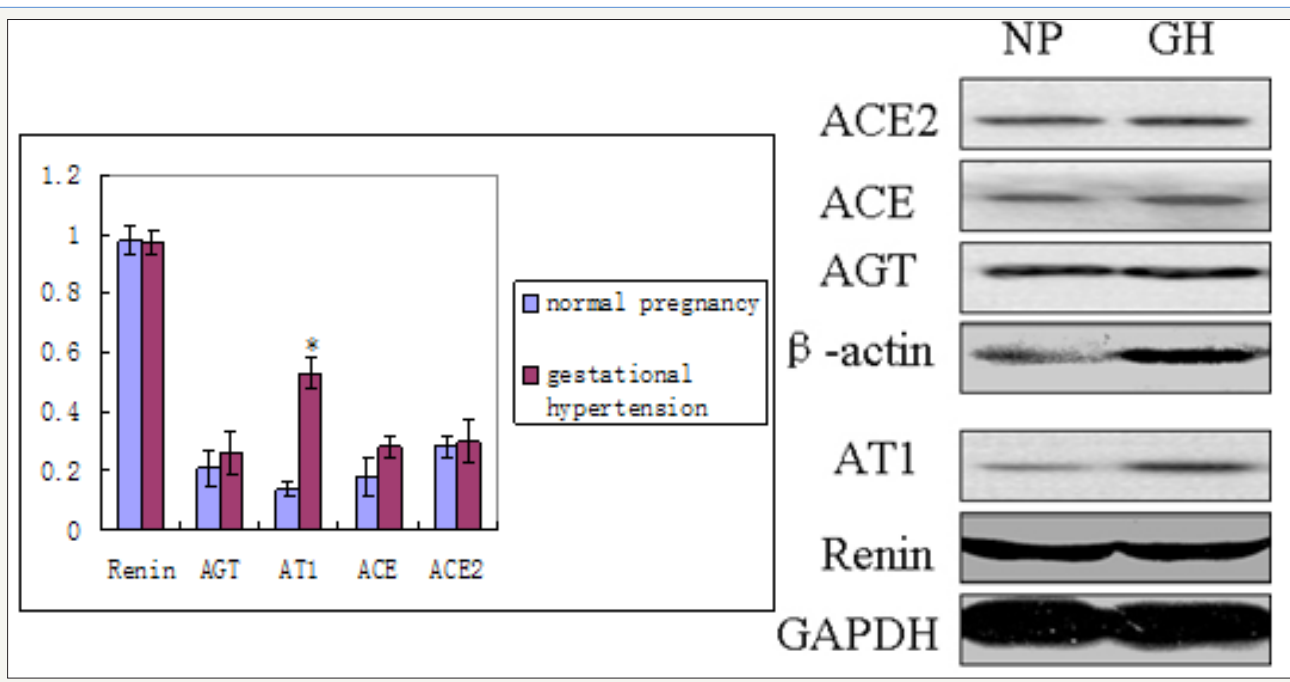

Figure 2b: RAS components protein of renin, AGT, angiotensin II receptor 1 (AT1), ACE, ACE2 were measured by western blot. NP: normal pregnancy GH: gestational hypertension.

\begin{tabular}{|c|c|c|c|c|}
\hline Unit: E-02 & Rrenin & AGT & AT1 & ACE \\
\hline Normal Pregnancy & $98 \pm 5$ & $21 \pm 6$ & $14 \pm 2$ & $28 \pm 3$ \\
\hline Gestational Hypertension & $97 \pm 4$ & $26 \pm 7$ & $53 \pm 5$ \\
\hline
\end{tabular}

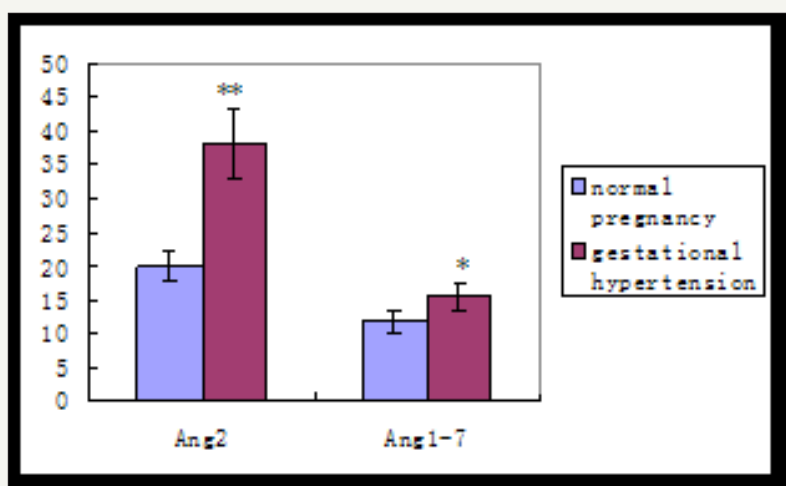

Figure 2c: RAS components protein of Ang 2 and Ang 1-7 were measured by ELISA.

\begin{tabular}{|c|c|c|}
\hline & Ang 2 & Ang 1-7 \\
\hline Normal Pregnancy & $20.02 \pm 2.07$ & $11.63 \pm 1.83$ \\
\hline Gestational Hypertension & $38.15 \pm 5.03$ & $15.44 \pm 2.03$ \\
\hline
\end{tabular}




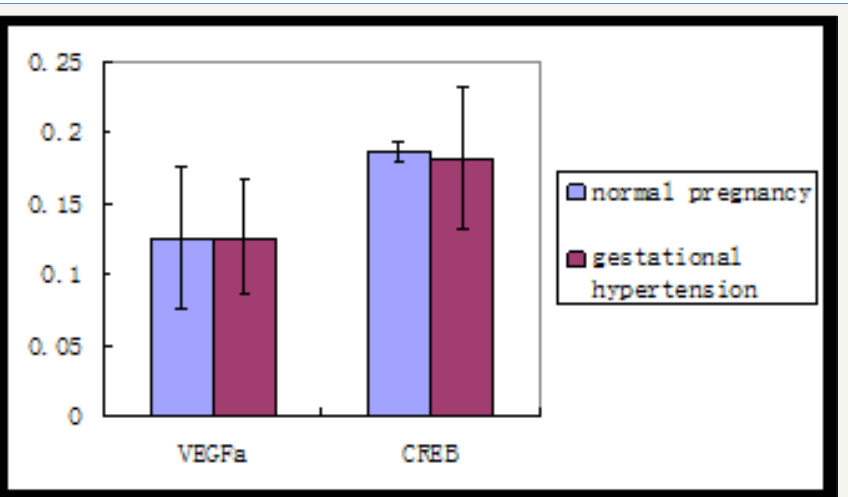

Figure 3: Gene expression of VEGFa and CREB in normal pregnant and gestational hypertension placenta by reverse transcription, real-time PCR.

\section{VEGFa and renin related gene, CREB expression in placenta and kidney}

Gene expression of VEGFa and CREB in normal pregnant and gestational hypertension placenta was determined by reverse transcription, real-time PCR. No significant difference was found in both of the genes mRNA between the two subjects. In addition, as shown in Figure 4a normal pregnant placenta versus gestational hypertension, no significant difference was found in the CREB protein expression. Besides that, CREB expression was also detected in human kidney tissues. Human kidney tissues are got from patients suffered with renal tumor or chronic renal insufficiency (CRI). Renal tumor with normal blood pressure is designed as control. A few CREB is found expressed at JG area. Compared with control, there are two results about the level of CREB staining in the
CRI kidney tissues, more concentration of CREB immune staining at JG area in one set (CRI group 1), and similar concentration of CREB expression in the other set (CRI group 2) compared with control (Figure $4 \mathrm{~b}$ ). Radioactive immunoassay was used to measure the renin activity in the circulating blood, as shown in Figure $2 b$, the set of high expression of CREB (CRI group 1) mean of plasma renin activity (PRA) increased significantly in comparison with the control, same result has been found in Angiotensin II (Ang II), Ang II increased correspondingly in CRI group 1. However, no statistical difference of the level of PRA and AngII has been found between CRI 2 and control. Data of blood pressure has shown that BP is higher than normal standard (120-140/69-90 $\mathrm{mmHg}$ ) in both of the two groups of CRI (Figure 4b) Table 2.

\begin{tabular}{|c|c|c|}
\hline & VEGFa & CREB \\
\hline Normal Pregnancy & $0.1248 \pm 0.05$ & $0.1864 \pm 0.04$ \\
\hline Gestational Hypertension & $0.1262 \pm 0.007$ & $0.1816 \pm 0.05$ \\
\hline
\end{tabular}

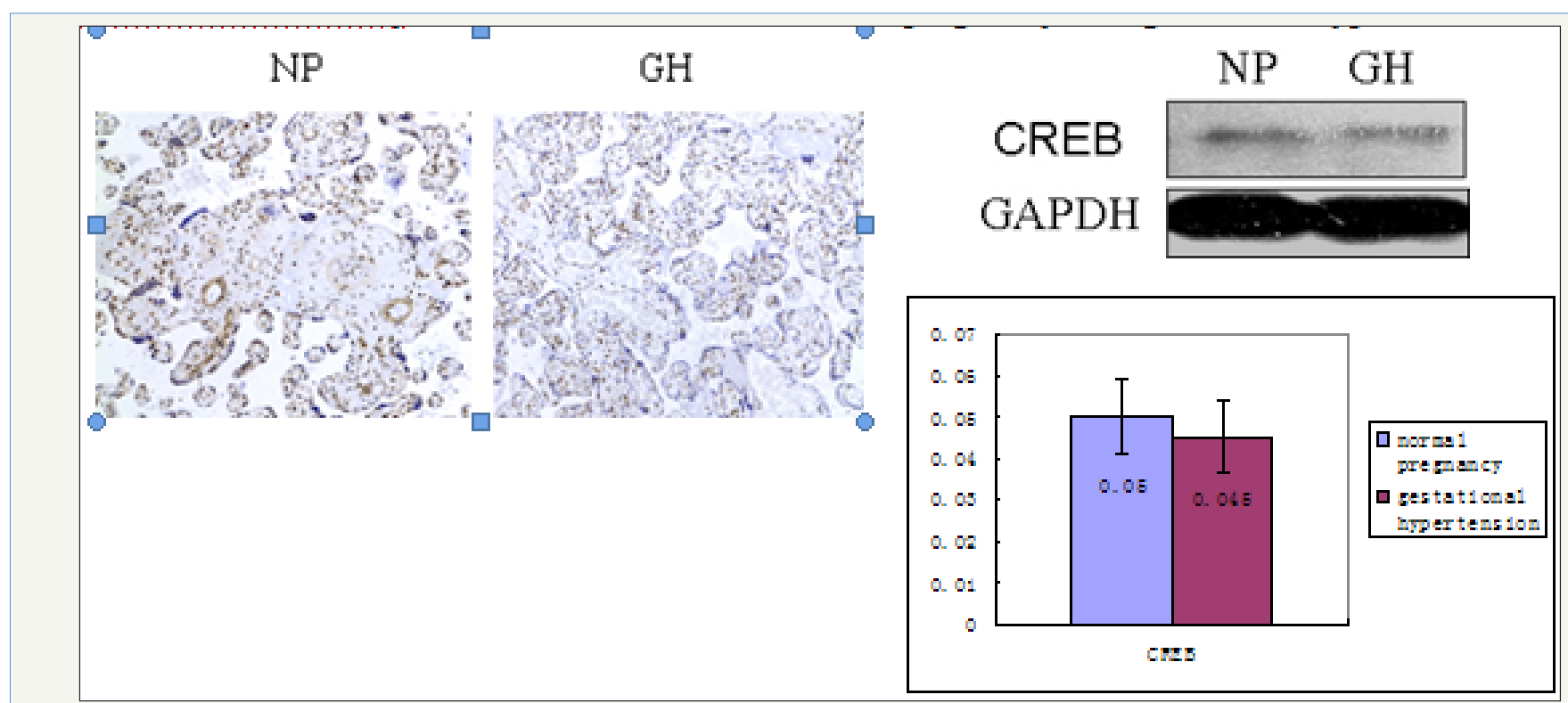

Figure 4a: Expression of CREB protein in placenta and kidney.

CREB expression detected in normal pregnant and gestational hypertension placenta with Immunohistochemistry and western blot NP: normal pregnancy GH: gestational hypertension. 

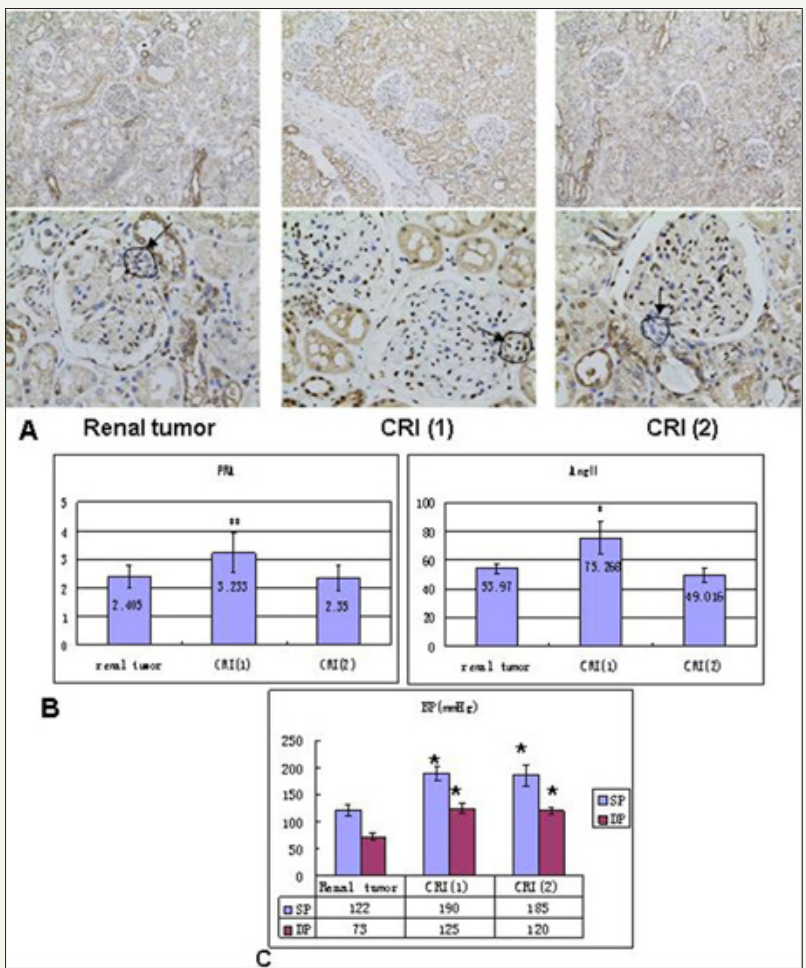

Figure 4b: CREB involved in renin expression in human kidney

A. Immunostaining for CREB in human kidneys from renal tumor, CRI (1) and CRI (2) respectively, figures with high magnification show in JG area more staining of CREB (brown nuclear) in CRI (1) than renal tumor and CRI (2), similar staining of CREB is in the latter two groups. B. Radioactive immunoassay show in the circulating blood renin activity and Ang II of CRI (1) are higher than renal tumor and CRI (2) significantly, no difference of renin activity and Ang II between renal tumor and CRI (2). C. Data of blood pressure show BP of CRI (1) and CRI (2) is higher compared with renal tumor, no difference between CRI (1) and CRI (2), $\mathrm{BP}$ of renal tumor is normal. CRI: chronic renal insufficiency PRA: plasma renin activity Ang II: Angiotensin II BP: blood pressure SP: systolic pressure DP: diastolic pressure ${ }^{*} \mathrm{P}<0.05$ vs renal tumor, ${ }^{* *} \mathrm{P}<0.01$ vs renal tumor

Table 2: Primers used for real-time PCR.

\begin{tabular}{|c|c|c|}
\hline & Gene & Primer Sequence \\
\hline \multirow{2}{*}{ CREB } & Forward & 5' CAAATGGAAAGGAAGGATAATGT 3' \\
\hline & Reverse & 5' CCAAAGAAACAGGAAGCAGATT 3' \\
\hline \multirow{2}{*}{ AGT } & Forward & 5' GCTTTCAACACCTACGTCCACT 3' \\
\hline & Reverse & 5' GGCACTTGAGTCACCGAGAA 3' \\
\hline \multirow{2}{*}{ Renin } & Forward & 5' GGCTCCTGTACCTTTGGTCT 3' \\
\hline & Reverse & 5' GTGGTGTTGCCAAGTGTCAG 3' \\
\hline \multirow[b]{2}{*}{ ACE } & Forward & 5' GGGACTTCTACAACGGCAA 3' \\
\hline & Reverse & 5' CTTGGGCGTAGACACTGAGA 3' \\
\hline \multirow{2}{*}{ ACE2 } & Forward & 5’ AGGATGTGCGAGTGGCTAAT 3' \\
\hline & Reverse & 5' TGGCTGTATCCCCAGAAACT 3' \\
\hline \multirow{2}{*}{ AT1 } & Forward & 5' GCCCTAAAGAAGGCTTATGAA 3' \\
\hline & Reverse & 5' CAGTCACGTATGATGCCTAGTTG 3' \\
\hline \multirow{2}{*}{ VEGFa } & Forward & 5' GAGGGCAGAATCATCACGAA 3' \\
\hline & Reverse & 5' GCACACAGGATGGCTTGAA 3' \\
\hline \multirow{2}{*}{ GAPDH } & Forward & 5' AACATACCAGATCCGCACAGCACC 3' \\
\hline & Reverse & 5' GTTCTTCATTAGACGGACCTCTCTCTTCCG 3 \\
\hline
\end{tabular}

\section{Discussion}

Hypertension is the most common medical disorder of pregnancy, which includes a spectrum of conditions, most notably preeclampsia, a form of hypertension unique to pregnancy that results in substantial maternal and neonatal morbidity and mortality. The other form, gestational hypertension, usually has more benign courses. Most study has concentrated on preeclampsia and few on gestational hypertension. Our research focus on the mechanism of gestational hypertension before preeclampsia occurred. Blood pressure increasing without proteinuria is the only symptom which happened in the subjects of gestational hypertension.

In an uncomplicated pregnancy, most circulating RAS components increase, however it is not the case in preeclampsia. Preeclamptic women have lower circulating levels of RAS components than their normal pregnant counterparts do [5]. The similar case has been found in gestational hypertension. Before delivery, both circulating active renin (PRL) and Ang II are lower than normotensive pregnant. In addition, we noticed that after delivery, PRL and Ang II decreased in the normal pregnant women, and Ang II has decreased significantly as well, while in the gestational hypertension women, both of PRL and Ang II increased. That can explain partly that no significant difference in PRL and Ang II are detected between normal pregnant and gestational hypertension after delivery. 
Since the first-time pro-renin, AGT, ACE, Ang II and Ang I, AT1 were identified in fetal placental tissues in 1998 by Li et al. [20], all the necessary components of RAS have been found both in maternal decidua and fetal placental tissues in succession [21]. However, the levels of RAS components in pre eclamptic placental were reported inconsistently $[5,21,22]$. The RAS plays an important role in placental development and regulation of utero placental blood circulation. In early pregnancy, Ang II promotes decidualization and rapid trophoblast proliferation mainly by binding to its receptor, AT1 [23]. In mid and late pregnancy, ACE, ACE2 and Ang (1-7) were elevated, which correlate with expanded maternal blood space and progressive fetal capillary development $[10,24,25]$. The dysregulation of RAS in placenta is associated with preeclampsia and other pathophysiological conditions during pregnancy.

Our study is the first to demonstrate the expression of RAS components in human gestational hypertension placental tissues. The classic system involves primarily two enzymes: renin and ACE. Renin mRNA was found to be present in normal and gestational hypertension placenta, besides that, no significant difference of its mRNA and protein levels was found in normal versus gestational hypertension, which is consistent with some previous reports in normal versus pre eclamptic [22]. On the contrary, in the study of Singh et al. [26] total renin concentration and active renin were significantly higher in preeclamptic placentas. The different results may indicate a possible trend for increased renin mRNA in the development from gestational hypertension to preeclamptic. ACE functions to hydrolyze Ang I to Ang II, and as its homolog, ACE2 degrade Ang II to Ang (1-7). The interplay between ACE and ACE2 may govern the formation and metabolism of Ang effector peptides. No statistical difference was seen in the levels of ACE and ACE2 between normal and gestational hypertension, which is consistent with most previous studies $[8,22,27]$, except the study of Ito et al, which reported significant increases in ACE mRNA and protein in the preeclamptic placenta. Reasons behind the difference are unclear. The presence of renin, ACE, ACE2 indicates their roles in both normal and gestational hypertension pregnancies.

The rate-limiting step of RAS cascade is the cleavage of AGT by renin to Ang I, a ten amino acid peptide without any biological function. Then Ang I is cleaved to Ang II, a biologically active eight amino acid molecule by ACE. Ang II, binding with its receptor AT1, is regarded as a well-known versatile effector involved in vasoconstriction, sympathetic activity, cellular growth and oxidative stress. Ang II can be cleaved to Ang (1-7) by ACE2. Ang (1-7) is considered to have a counteracting effect to Ang II on the cardiovascular system by its inhibition of cell migration and proliferation $[5,21,28]$. Expression of AGT was found in our study, and no significant difference was found in the placenta of normal versus gestational hypertension, which was consistent with the study of Herse et al. [8]. However, in the study of Lauren et al, AGT mRNA was higher in the chorionic villi obtained from preeclamptic subjects [22]. The conflicting investigation may result from the tissues studied, what Lauren et al used was exclusively chorinonic villi, while what Herse et al. [8] and we used was whole placenta. Another reason that account for the confliction with Lauren et al is our subjects are gestational hypertension, having not developed to preeclampsia yet.

A number of studies have measured the receptor of Ang II, AT1, in the placenta of women with normal and preeclamptic pregnancies, but there is no agreement yet. The conflicting reports include AT1 was upregulated [29], downregulated [30], or not different [8]. It should be noticed that the subjects in these studies are preeclampsia. In our study, the level of AT1 mRNA in the placenta of gestational hypertension is higher than that in normal pregnancy, although there is no statistical difference between them. The level of AT1 protein was found more than 3-fold upregulated in gestational hypertension versus normal. The mismatches of mRNA and protein measurements have been revealed by numerous studies. Consideration should be given to other factors involved in the translation of AT1 gene.

We also found that Ang II was around twice as high in the placenta of gestational hypertension when compared with normal pregnancy, which is same as the result of [22]. And what interesting is that circulating Ang II was lower in the gestational hypertension. In addition, circulating active renin was lower in the gestational hypertension too, while in the placenta, no difference was found between gestational hypertension and normal. The difference between in circulating and in local placenta suggests redistribution of RAS components, and that contribute to vasoconstriction of the fetal blood vessels and decrease of maternal-fetal transport. Besides that, in contrast with previous studies on the preeclampsia that no difference in Ang (1-7) expression was found versus normal pregnancy [22], or even reduction in Ang (1-7) expression was detected versus normal pregnancy [25,31], we found that in the placenta of gestational hypertension, Ang (1-7) is significantly higher than normal control. It means that at the beginning and benign stage of hypertension during pregnancy, local RAS has been regulated by increasing the expression of Ang (1-7) to try to modulate the effects of RAS on vascular tone, neutralizing excessive contraction. Ang (1-7) counteract the reduction of Ang II in placental blood flow by interacting with AT1. Expression of AT1 protein in the placenta of gestational hypertension is greatly higher than the normal subject can be explained to meet the increasing of Ang II and Ang (1-7). RAS has become a "system within a system" [32,33], Not only ACE-mediated arm of the RAS must be considered, but the ACE2 pathway, giving rise to the vasodilatory and anti proliferative peptide Ang (1-7), should also be put attention to.

It is interesting that in contrast to the significant increase in Ang II and Ang (1-7), no change in ACE and ACE2 levels were found between gestational hypertension and normal control. It suggests that the system is being driven by other regulatory factors. Consideration should also be given to other genes involved in the production of RAS components.

Vascular endothelial growth factor (VEGF) is one of important factors produced from the human placenta, which plays a key role in promoting angiogenesis [34]. Ang II can induce the over expression of VEGF by acting through the AT1 $[35,36]$. While in our study, no difference of VEGFa mRNA was found in gestational hypertension 
versus normal pregnancy. The result is consistent with previous study in preeclampsia that VEGF release was unchanged by Ang II and Ang (1-7) [37]. The mechanism of angiogenesis in the placenta needs to be explored more.

CREB is known to mediate the stimulation of renin expression, and determine the basal transcription rate of the renin gene [38]. Ang II can lead to the activation of CREB through multiple signaling pathways activating. CREB feed back to upregulate the AT1 gene [39]. In our study on human kidney tissue, we found that the mechanism of all kinds of hypertensive cases is different and complicated. In the chronic renal insufficiency patients, some mechanisms of hypertension are independent of the circulating RAS, and some are caused by renin increased. More CREB was detected at the JG area where renin was expressed in the circulating PRL increased subjects, but not in that no change of PRL subjects. The data shows that renin increased by CREB is one of the causes for high BP in chronic renal insufficiency. However we did not find any change of CREB expression in the placenta between the gestational hypertension and normal pregnancy, combined with no change of renin expression in placenta either. It seems that regulation on the local RAS in placenta is independent in CREB and renin. It is necessary to conduct further investigation.

\section{Conclusion}

This study provides evidence for the presence and regulation of a local tissue-specific RAS in the benign stage of hypertension during pregnancy, gestational hypertension. Besides of the increase of Ang II and AT1, which is consistent with many other previous reports in preeclampsia, we found that in gestational hypertension local Ang (1-7) level was significantly higher than normal control. The change of Ang (1-7) expression is different from the studies in preeclampsia. Ang II and Ang (1-7), the two active peptides of the RAS in the placenta, their balance contain the fetal vessels and make up the cell barrier between maternal and fetal blood. The major actions of Ang II may be contributing to the pathophysiology of gestational hypertension, and Ang (1-7) has counteracting effect to Ang II. Increase of Ang (1-7) in the stage of gestational hypertension is the regulation of local RAS, when the balance is broken, the disease would develop into preeclampsia. Because the common factors, CREB and VEGFa, involving in the hypertension and angiogenesis were not found to be the cause for the hypertension during pregnancy, the mechanism of gestational hypertension needs to be investigated more. In summary, hypertension during pregnancy is characterized by a series of significant alterations in RAS worthy of deeper research. The finding of Ang (1-7) increasing in gestational hypertension may lead to a new therapy for diseases.

\section{References}

1. Reem M, Sana A, Anu G, Rocco CV (2012) A Comprehensive Review of Hypertension in Pregnancy. J Pregnancy.

2. Almundher AI-M, Asnat W, Gideon K (2012) Taking angiotensinconverting enzyme inhibitors during pregnancy, is it safe? Can Fam Physician 58(1): 49-51.

3. Duley L (2009) The global impact of pre-eclampsia and eclampsia. Semin Perinatol 33(3): 130-137
4. Langer B, Grima M, Coquard C, Bader AM, Schlaeder G, Imbs JL (1998) Plasma active renin, angiotensin I, and angiotensin II during pregnancy and in preeclampsia. Obstet Gynecol 91(2): 196-202.

5. Roxanna AI, Yang X (2011) Renin angiotensin signaling in normal pregnancy and preeclampsia. Semin Nephrol 31(1): 47-58.

6. Shah DM (2006) The role of RAS in the pathogenesis of preeclampsia. Curr Hypertens Rep 8(2): 144-152.

7. Shah DM, Banu JM, Chirgwin JM, Tekmal (2000) Reproductive tissue renin gene expression in preeclampsia. Hypertens Pregnancy 19(3): 341-351.

8. Herse F, Dechend R, Harsem NK, Wallukat G, Janke J, Qadri F, Hering L, Muller DN, Staff AC (2007) Dysregulation of the circulating and tissuebased rennin-angiotensin system in preeclampsia. Hypertension 49(3): 604-611.

9. Anton L, Brosnihan BK (2008) Systemic and uteroplacental renninangiotensin system in normal and preeclampsia pregnancies. Ther Adv Cardiovasc Dis 2(5): 349-362.

10. Neves L, Stovall K, Joyner J, Valdes G, Gallagher PE, Ferrario CM, Merrill DC, Brosnihan KB (2008) ACE2 and ANG (1-7) in the rat uterus during darly and late gestation. Am J Physiol Regul Integr Comp Physiol 294(1):151-161.

11. Sequeira Lopez ML, Gomez RA (2010) Novel mechanisms for the control of renin synthesis and release. Curr Hypertens Rep 12(1): 26-32.

12. Pentz ES, Sequeira Lopez ML, Cordaillat M, Gomez RA (2008) Identity of the renin cell is mediated by cAMP and chromatin remodeling: an in vitro model for studying cell recruitment and plasticity. Am J Physiol Heart Circ Physiol 294(2): H699-H707.

13. Arany Z, Newsome D, Oldread E, Livingston DM, Eckner R (1995) A family of transcriptional adaptor proteins targeted by the E1A oncoprotein. Nature 374(6517): 81-84

14. Lundblad JR, Kwok RP, Laurance ME, Harter ML, Goodman RH (1995) Adenoviral E1A-associated protein p300 as a functional homologue of the transcriptional co-activator CBP. Nature 374(6517): 85-88.

15. Gomez RA, Ellen SP, Xuan J, Magali C, Maria LSL (2009) CBP and p300 are essential for renin cell identity and morphological integrity of the kidney. Am J Physiol Heart Circ Physiol 296(5): 1255-1262.

16. Pan L, Black TA, Shi Q Jones CA, Petrovic N, Loudon J, Kane C, Sigmund CD, Gross KW (2001) Critical roles of a cyclic AMP responsive element and an E-box in regulation of mouse renin gene expression. J Biol Chem 276(49): 45530-45538.

17. Taylor RN, Davidge ST, Roberts JM (2009) Endothelial Cell Dysfunction and Oxidative Stress in Chesley's Hypertensive Disorders in Pregnancy. In: Lindheimer MD, Cunningham FG, et al. (Eds.), ( $3^{\text {rd }}$ edn), The Netherlands, pp: 145-170.

18. Maynard SE, Min JY, Merchan J, Lim KH, Li J, et al (2003) Excess placental soluble fms-like tyrosine kinase 1 (sFlt 1) may contribute to endothelial dysfunction hypertension, and proteinuria in preeclampsia. J Clin Invest 111(5): 649-658.

19. The ARIC Investigators (1987) Sitting blood pressure and postural changes in blood pressure and heart rate. ARIC Manual 11, ARIC Coordinating Center, Department of Biostatistics, University of North Carolina. Chapel Hill, NC.

20. Li X, Shams M, Zhu J, Khalig A, Wilkes M, Whittle M, Barnes N, Ahmed A (1998) Cellular localization of AT1 receptor mRNA and protein in normal placenta and its reduced expression in intrauterine growth restriction. Angiotensin II stimulates the release of vasorelaxants. J Clin Invest 101(2): 442-454

21. Jie Y, Jianyu S, Suli Z, Hao L, Huirong L (2013) The role of the renin-angiotensin-aldosterone system in preeclampsia: genetic polymorphisms and microRNA. J Mol Endocrinol 50(2): 53-66. 
22. Lauren A, David CM, Liomar AAN, Kathryn S, Patricia EG, Debra ID, Cheryl M, Courtney G, Carlos MF, Bridget KB (2008) Activation of local chorionic villi antiotensin II levels but not angiotensin (1-7) in preeclampsia. Hypertension 51(4): 1066-1072.

23. Hering L, Herse F, Geusens N, Verlohren S, Wenzel K, et al (2010) Effects of circulating and local uteroplacental angiotensin II in rat pregnancy. Hypertension 56(2): 311-318.

24. Levy A, Yagil Y, Bursztyn M, Barkalifa R, Scharf S, et al (2008): ACE2 expression and activity are enhanced during pregnancy. Am J Physiol Regul Integr Comp Physiol 295(6): 1953-1961.

25. Haijun G, Uma Y, Chandra Y (2012) Maternal protein restriction reduces expression of angiotensin I-converting enzyme 2 in rat placental labyrinth zone in late pregnancy. Biol Reprod 86(2): 31.

26. Singh HJ, Rahman A, Larmie ET, Nila A (2004) Raised prorenin and renin concentrations in pre-eclamptic placentae when measured after acid activation. Placenta 25(7): 631-636.

27. Kalenga MK, Thomas K, deGasparo M, De H (1996) Determination of renin-angiotensin converting enzyme and angiotensin II levels in human placenta, chorion and amnion from women with pregnancy induced hypertension. Clin Endocrinol (Oxf) 44(4): 429-433.

28. Wutao Z, Weiyan C, Xiuyu L, Jiangui H, Hong M (2009) Chronic angiotensin-(1-7) administration improves vascular remodeling after angioplasty through the regulation of the TGF- $\beta /$ Smad signaling pathway in rabbits. Biochem Biophys Res Commun 389(1): 138-144.

29. Leung PS, Tsai SJ, Wallukat G, Leung TN, Lau TK (2001) The upregulation of angiotensin II receptor AT(1) in human preeclamptic placenta. Mol Cell Endocrinol 184(1): 95-102.

30. Knock GA, Sullivan MH, McCarthy A, Elder MG, Polak JM, Wharton (1994) Angiotensin II (AT1) vascular binding sites in human placenta from normal-term, preeclamptic and growth retarded pregnancies. Pharmacol Exp Ther 271(2): 1007-1015.
31. Merrill DC, Karoly M, Chen K, Ferrario CM, Brosnihan KB (2002) Angiotensin-(1-7) in normal and preeclamptic pregnancy. Endocrine 18(3): 239-245

32. Yagil Y, Yagil C (2005) Hypothesis: ACE2 modulates blood pressure in the mammalian organism. Hypertension 41(4): 871-873.

33. K.Bridget B, Liomar AAN, Mark CC: (2005) Does The AntiotensinConverting Enzyme (ACE)/ACE2 Balance Contribute to the Fate of Antiotensin Peptides in Programmed Hypertension? Hypertension 46: 1097-1099.

34. Brosnihan KB, Bharadwaj MS, Yamaleyeva LM, Neves LAA (2012) Decidualized pseudopregnant rat uterus shows marked reduction in Ang II and Ang-(1-7) levels. Placenta 33(1): 17-23.

35. Amaral SL, Linderman JR, Morse MM, Greene AS (2001) Angiogenesis induced by electrical stimulation is mediated by angiotensin II and VEGF. Microcirculation 8(1): 57-67.

36. Vitoratos N, Hassiakos D, Iavazzo C (2012) Molecular mechanisms of preeclampsia. J Pregnancy.

37. Lauren A, David CM, Liomar AAN, Courtney G, Cheryl M, et al. (2010) Angiotensin II and angiotensin-(1-7) decrease sFlt1 release in norma but not preeclamptic chorionic villi: an in vitro study. Reprod Biol Endocrinol 8: 135-145.

38. Hayo C, Klaus H, Armin K, Frank S, Vladimir T, Charlotte W (2010) Physiology of kidney renin. Physiol Rev 90(2): 607-673.

39. Karia KVH, Amit M, Irving HZ (2013) NF-kB and CREB Are Required for Angiotensin II Type 1 Receptor Upregulation in Neurons. Plos One 8(11): e78695.
Creative Commons Attribution 4.0 International License

For possible submissions Click Here

\section{Submit Article}

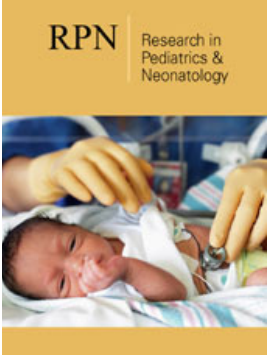

\section{Research in Pediatrics \& Neonatology}

\section{Benefits of Publishing with us}

- High-level peer review and editorial services

- Freely accessible online immediately upon publication

- Authors retain the copyright to their work

- Licensing it under a Creative Commons license

- Visibility through different online platforms 